\title{
Multilayer contacts in electrical connectors: experimental results and modelling
}

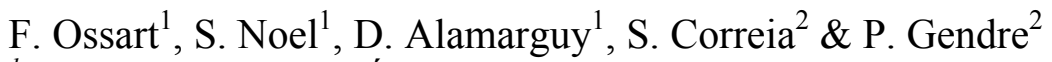 \\ ${ }^{1}$ Laboratoire de Génie Électrique de Paris, Supélec, \\ Universités Paris VI et Paris XI, Gif sur Yvette, France \\ ${ }^{2}$ PEM, PEM Siaugues Saint Romain, Siaugues Sainte Marie, France
}

\begin{abstract}
Electrical contacts are an essential part of electrical circuits and many reliability problems are related to their failure. The present work uses numerical simulation in view of a better analysis of the electromechanical phenomena, in the case of multilayer electrical contacts. We study a ball/plane contact made of bulk CuZn alloy, protected by a thin Sn surface layer. A coupled finite element analysis is performed in order to calculate the contact resistance of the device: an elasto-plastic model is used to determine the geometry of the contact area, then an electrical model gives the resulting constriction resistance. Results of the simulation are compared to experimental data. The respective contributions of the mechanical and electrical phenomena are analysed.

Keywords: multilayer contact, finite element modelling, constriction resistance, tin coating, electric contact.
\end{abstract}

\section{Introduction}

Electrical connector performances are closely linked to the mechanical behaviour of the contact. Much work has been devoted to understand the mechanisms involved and their complexity has been shown [1, 2]. Many contacts are made of cuprous alloys with various plating layers. Tin finishes are commonly used because they are cheap and can protect fairly well the copper substrates. This study is part of a larger one aimed at investigating and improving the properties of electroplated tin layers [3]. The contacts under study are of the ball plane type; their geometry simulates elementary contacts in connectors. They are submitted to a normal load and to the various failure tests representative of their conditions 
of use. Various platings are shown to display different properties; the influence of the tin thickness is particularly important. When brass $(\mathrm{CuZn})$ is used for the substrate, a nickel underlayer is often deposited in order to prevent diffusion of zinc at the surface. Thus we have investigated the influence of such an underlayer on the contact behaviour.

Electrical contacts are characterised by their contact resistance. This global parameter takes into account both mechanical and electrical phenomena: its calculation requires a coupled analysis. We describe here the first results.

\section{Experimental results}

Samples are brass ( $\mathrm{Cu} 70 \mathrm{Zn} \mathrm{30)}$ coupons with a layer of tin electrodeposited from a bright or matte bath. The properties of the materials used for samples are described in table 1. Tin baths are based on MSA (methane sulfonic acid) chemistry. An underlayer of matte nickel was deposited on some of the coupons. Series of brass coupons were coated with tin layers of various thicknesses from $0.1 \mu \mathrm{m}$ to $10 \mu \mathrm{m}$. Particular care was taken in order to have reproducible and constant values of the tin thickness. Micro-hardness measurements were performed with a Leitz Miniload 2 apparatus on the cuprous substrates either bare or with especially thick $(50 \mu \mathrm{m}$ to $100 \mu \mathrm{m})$ layers of the two types of tin electrodeposits. The bright finish tin layer has a higher value of hardness than the matte tin finish. The experimental yielding stress values $\sigma_{Y}$ of table 1 are deduced from these microhardness measurements. A sphere of $1.3 \mathrm{~mm}$ of radius was stamped in some of the flats.

Several special devices (described in [4] for example) allowed the simulation of the degradation mechanisms leading to connector failure. The effect of mating and unmating connectors was simulated with a reciprocating wear test (constant speed $0.5 \mathrm{~mm} / \mathrm{s}$, amplitude $2 \mathrm{~mm}$; normal load $250 \mathrm{gf}$ ). The contact resistance $R c$ values were measured in static and then during the friction test, at the end of each wear track with a four wire method with $+/-20 \mathrm{~mA}$ in order to eliminate thermal fem. A dedicated device was used to study the evolution of the ball/plane contact resistance during a fretting test (small amplitude oscillatory movements simulating the effect of vibrations): with the following parameters: normal load $250 \mathrm{gf}$, amplitude $50 \mu \mathrm{m}$ and frequency $1 \mathrm{~Hz}$.

These tests allow the characterisation of the properties of platings for the application. Figure 1 shows the evolution of $R c$ (in static) for substrates coated with matte tin and bright tin layers of increasing thickness. It also shows the resistance values obtained with a nickel underlayer between $\mathrm{CuZn}$ and tin. $R c$ values lay between $0.8 \mathrm{~m} \Omega$ and $2 \mathrm{~m} \Omega$. They decrease with increasing tin thickness. The nickel underlayer has no effect for bright coatings but causes the resistance values to increase for matte tin ones.

When the contacts are submitted to mechanical tests (wear or fretting) very different behaviours can be recorded for the various platings. An example is shown in Fig. 2 where we have plotted the number of cycles (of amplitude 50 $\mu \mathrm{m})$ after which the electrical properties are degraded (when the contact resistance values reach $10 \mathrm{~m} \Omega$ ). 


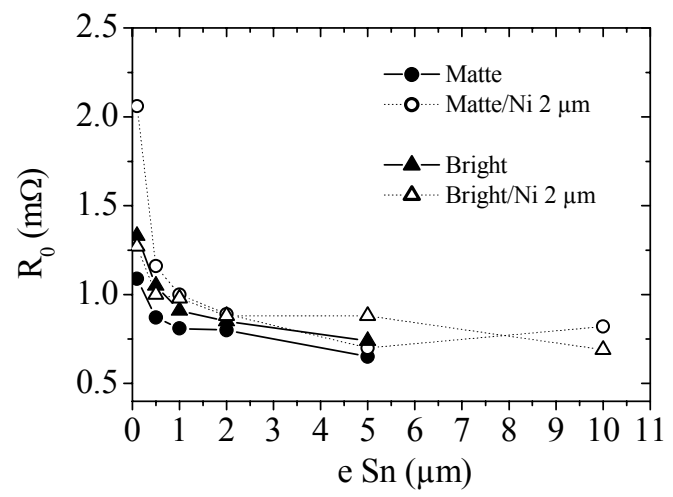

Figure 1: Experimental contact resistance values $(\mathrm{F}=250 \mathrm{gf})$ for bright and matte tin layers on $\mathrm{CuZn}$ substrate or $\mathrm{CuZn}$ with a nickel underlayer, for various tin coating thicknesses.

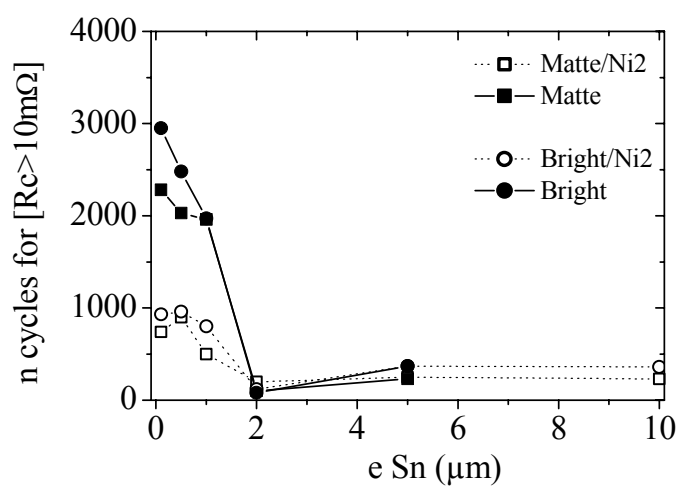

Figure 2: Number of "fretting" cycles $(\mathrm{F}=250 \mathrm{gf}, \mathrm{a}=+/-25 \mathrm{~mm}, \mathrm{f}=1 \mathrm{~Hz})$ at which $\mathrm{Rc}>10 \mathrm{~m} \Omega$ for different tin platings and different tin layer thicknesses.

\section{Finite element model}

The contact resistance which characterises electrical contacts is a global parameter resulting from both mechanical and electrical phenomena: hence, its calculation requires a coupled analysis. In the case of multilayered contacts submitted to plastic deformations, simple analytical models are not available and a numerical approach is needed. The finite element method is well suited to account for the geometry of the device and for the non-uniform mechanical and electrical properties of the layers. Only a weak coupled analysis is required: the contact resistance depends on the mechanical behaviour via the geometry of the contact area, but conversely the mechanical state of the device is not affected by 
its electrical behaviour. This corresponds to the case of low level electrical contacts where no thermal effects occur. Hence, we do a mechanical analysis, simply followed by an electrical one for a static contact. The commercially available finite element software ANSYS Multiphysics [5] was used.

\subsection{Mechanical analysis}

The ball/plane contact is an axi-symmetric problem and a 2d-model can be used. An elasto-plastic finite element analysis accounting for large deformations and contact is performed. The geometry, boundary conditions and loading are shown on Fig. 3. The contact between the upper and lower parts of the contact is managed by ANSYS, which determines which face-to-face elements come into contact for a given load. The radius of the contact area is computed for increasing loads up to $300 \mathrm{gf}$, and for Sn thickness between 0 and $10 \mu \mathrm{m}$. 6-node triangular elements are used and special care was taken to properly mesh the layers and the contact area. Due to the meshing, the contact radius increases discontinuously with F, whenever two new elements come into contact. Two assumptions are made. First we suppose that there is no friction between the two parts of the contact and secondly that there is perfect adhesion of the tin layer on the substrate. The material 'stress-strain' behaviours are represented by bilinear curves, characterised by the elastic Young modulus E for stresses below the yielding stress $\sigma_{\mathrm{Y}}$, and a hardening coefficient $\gamma=d \sigma / d \varepsilon$ above. The bulk part of the contact is made of a CuZn alloy. We model two types of Sn-layers with different hardness values, referred to as "soft" and "hard" Sn hereafter. The influence of a $2 \mu \mathrm{m}$-thick Ni underlayer was also studied.

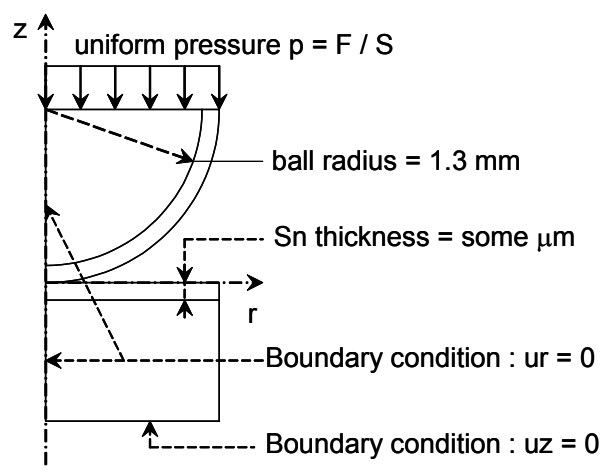

Figure 3: Geometry and boundary conditions of the 2d-axisymmetric model.

The characteristics of the materials are summarised in table 1 .

In a first stage, the validity of the model is checked, by comparing with the Hertz model which provides an analytical solution for perfect contacts (no friction and homogeneous elastic materials). Formula (1) gives the Hertz contact radius $a$. Parameters $R, F, E$ and $v$ are respectively for the ball radius, the applied force, the Young modulus and the Poisson coefficient of the material [7]. 
Table 1: Mechanical and electrical characteristics of the materials used. (a) manufacturer data, (b) measured data, (c) Hammam [6].

\begin{tabular}{lccccl}
\hline & $\mathrm{E}(\mathrm{GPa})$ & $\sigma_{\mathrm{Y}}(\mathrm{MPa})$ & $\gamma(\mathrm{GPa})$ & $v$ & $\rho(\Omega \mathrm{m})$ \\
\hline $\mathrm{CuZn}$ & $114^{(\mathrm{a})}$ & $370^{(\mathrm{b})}$ & $0.55^{(\mathrm{a})}$ & $0.34^{(\mathrm{a})}$ & $6.2 .10^{-8(\mathrm{a})}$ \\
\hline Sn matte & 50 & $25^{(\mathrm{b})}$ & 0.55 & 0.34 & $26.5 .10^{(\mathrm{c})}$ \\
\hline Sn bright & 50 & $70^{(\mathrm{b})}$ & 0.55 & 0.34 & $26.5 .10^{-8(\mathrm{c})}$ \\
\hline $\mathrm{Ni}$ & 220 & $>600^{(\mathrm{b})}$ & 0.55 & 0.34 & $6.9 .10^{-8(\mathrm{c})}$ \\
\hline
\end{tabular}

$$
a=\sqrt[3]{\frac{3}{4} \cdot \frac{F R}{E^{*}}} \quad \text { with } E^{*}=\frac{E}{2\left(1-v^{2}\right)}
$$

The finite element model should give the same results as Hertz model at low forces (elastic regime). But because the finite element model accounts for plasticity, it will give larger contact area after some critical load has been reached (elasto-plastic regime).

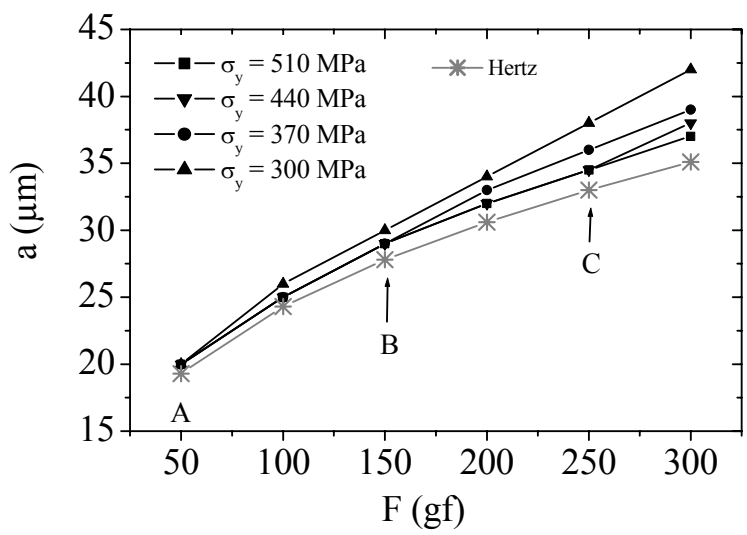

Figure 4: $\quad$ Contact radius a $(\mu \mathrm{m})$ vs. applied force $\mathrm{F}(\mathrm{gf})$, for different values of $\sigma_{\mathrm{Y}}: 510 \mathrm{MPa}, 440 \mathrm{MPa}, 370 \mathrm{MPa}$ (exp. value) and $300 \mathrm{MPa}$.

Different yielding stresses $\sigma_{Y}$ corresponding to various usual cuprous substrates are tested, so as to see how the critical force between the elastic and the elasto-plastic regime depends on this parameter (Fig. 4). For $\sigma_{Y}=510 \mathrm{MPa}$, the global behaviour remains elastic up to $3 \mathrm{~N}$. We note that the finite element model overestimates the contact radius. This systematic error (around $1 \mu \mathrm{m}$ ) is due to the mesh. Using smaller elements allows this error to diminish, but the computational cost is high and no significant changes of the global curves are observed. For lower values of $\sigma_{Y}$, the elastic regime ends for lower value of $\mathrm{F}$ (point $\mathrm{A}: \mathrm{F}=50 \mathrm{gf}$ for $\sigma_{\mathrm{Y}}=300 \mathrm{MPa}$, point $\mathrm{B}: \mathrm{F}=150 \mathrm{gf}$ for $\sigma_{\mathrm{Y}}=370 \mathrm{MPa}$ and point $\mathrm{C}: \mathrm{F}=250 \mathrm{gf}$ for $\left.\sigma_{\mathrm{Y}}=300 \mathrm{MPa}\right)$. We obtain critical values of $\mathrm{F}$ which are consistent with the 'rule of the thumb' given by formula (2).

$$
3 F_{y} \leq F_{\text {critical }} \leq 5 F_{y} \quad \text { where } F_{y}=21 \frac{R^{2} \sigma_{Y}^{3}}{E^{* 2}}
$$


The next step is to study the influence of the Sn-layer on the contact area. Figure 5 shows the contact radius as a function of the Sn-thickness, for applied forces ranging between $50 \mathrm{gf}$ and $300 \mathrm{gf}$, in the case of hard and soft Sn. The curves give expected continuous trends. The contact radius increases with the load. It also increases with the Sn thickness because this material is softer than the $\mathrm{CuZn}$ substrate. Larger contact areas are obtained with the softer Sn layer. An example of deformed $\mathrm{Sn}$ layer is shown on Fig. 6 (
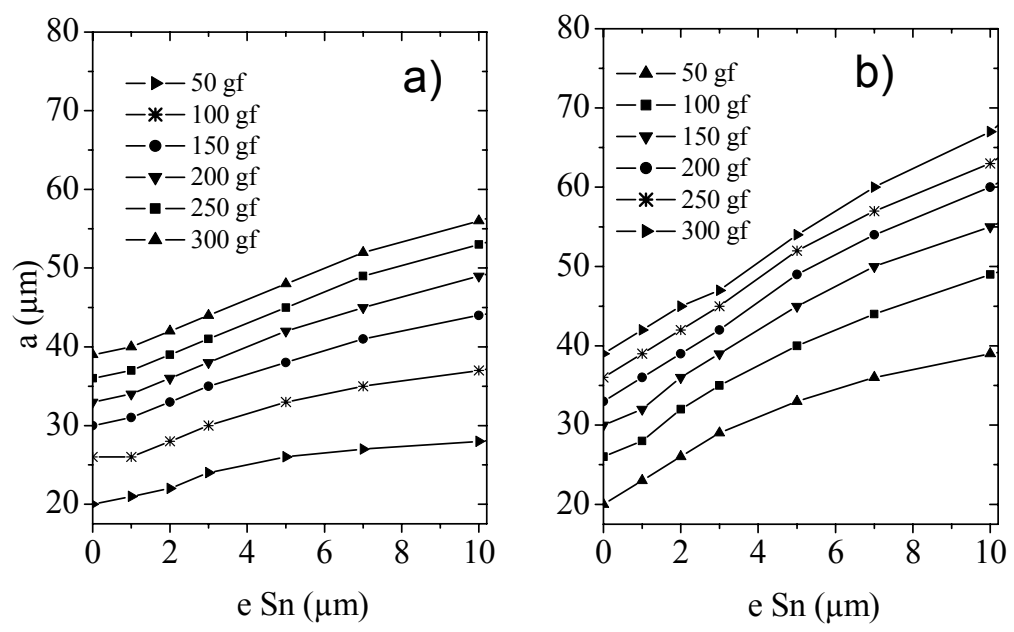

Figure 5: Contact radius a, versus Sn thickness e, for different applied forces. a): hard $\left.\operatorname{Sn}\left(\sigma_{Y}=70 \mathrm{MPa}\right)-b\right)$ : $\operatorname{soft} \operatorname{Sn}\left(\sigma_{Y}=25 \mathrm{MPa}\right)$.

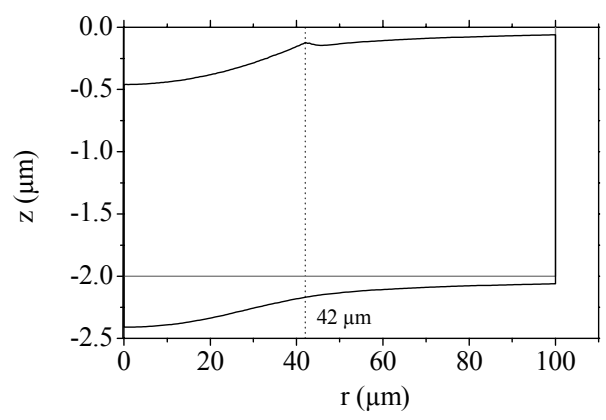

Figure 6: Geometry of the bottom $\mathrm{Sn}$ layer in the contact area $(2 \mu \mathrm{m})$.

The contact area spreads between the vertical axis and the dashed line $(r=42$ $\mu \mathrm{m})$. It is worth noticing that the geometry of the layer does not change significantly. There is a slight bending and, although the tin is very soft, its thickness remains the same. This behaviour is due to the perfect adhesion of the $\mathrm{Sn}$ film on the CuZn substrate, which prevents the radial displacement of Sn caused by the contact pressure. 


\subsection{Electrical analysis:}

The electrical model allows the global resistance of the contact to be calculated, for given geometry and electrical properties. Figure 7 shows the current lines in the contact area, when a difference of potential $\Delta V$ is applied between the top and bottom surfaces of the contact. Let us denote $I$ the resulting intensity. The contact resistance $R_{c}$ is defined as $R_{c}=\Delta V / I$. The small section of the contact area leads to a strong constriction of the current in this region and a high local resistance.

When two infinite electrodes touch at a single circular spot of radius $a$, the formula for the contact resistance is $R_{c}=\rho / 2 a[1,7]$. The contact radius is the only geometric parameter, because constriction is the dominant mechanism in the contact.

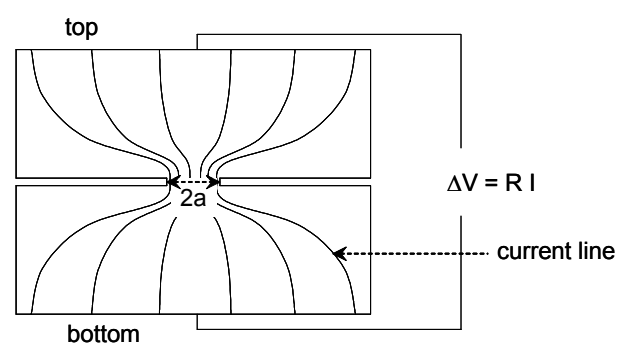

Figure 7: Constriction of the current lines in the contact area.

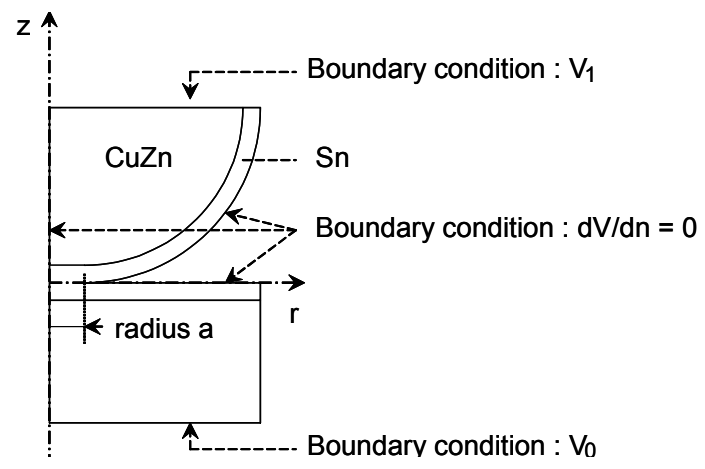

Figure 8: Geometry and boundary conditions of the finite element model.

In the case of a multilayer contact $R_{c}=\rho / 2 a$ does not hold and a numerical analysis is needed. Again, we use the finite element method to deal with the nonuniform properties of multilayer contacts. As we have seen, the results of the mechanical analysis indicate that the change of geometry of the Sn layer under the load is very small. Hence, we neglect it. We assume a perfect electrical contact between both sides of the contact. The radius of the contact area is the result of the mechanical analysis. Figure 8 summarises the problem solved. 
A difference of electric potential is applied between the top and the bottom of the contact, and the resulting current distribution is calculated. The total current $I$ is obtained by integration of the current density, and the global resistance of the contact $R c$ is given by: $R_{c}=\left(V_{1}-V_{0}\right) / I$.

$R c$ was calculated as a function of the Sn-layer thicknesses for all the applied forces. Since the value of the resistivity of thin tin layers found in literature could vary from 6 to $2610^{-8} \Omega . \mathrm{m}$, calculations were performed for these various values. Results are plotted for hard tin Fig. 9 a) and for soft tin Fig. 9 b). These figures also display the calculation of $R_{c}=\rho / 2 a$ (with $\rho$ the resistivity of the substrate $\mathrm{CuZn}$ and $a$ the contact radius calculated from the mechanical finite element FEM model) and the experimental values measured for the bright (hard) and matte (soft) tin layers on the CuZn substrate.
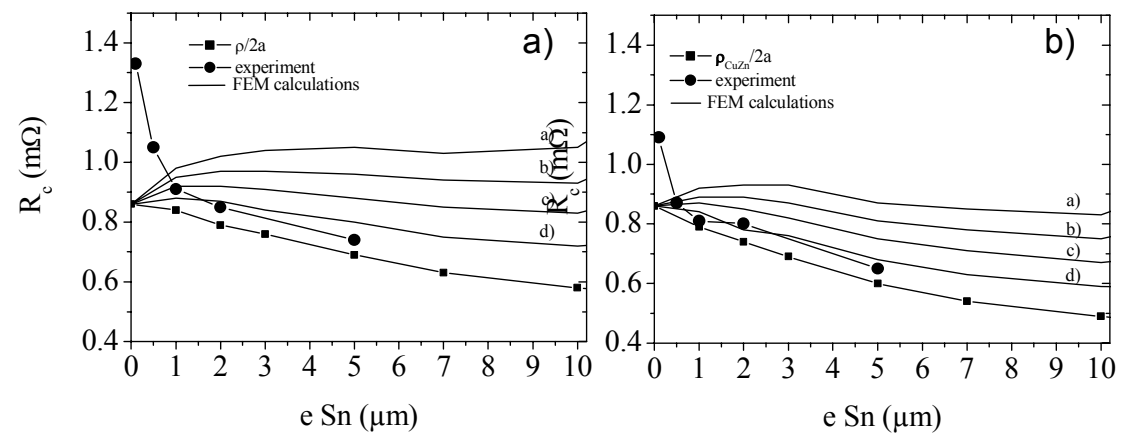

Figure 9: Contact resistance vs. Sn layer thickness, for different values of $\rho$ [a) $26.5 .10^{-8} \Omega \mathrm{m}-$ b) $21.10^{-8} \Omega \mathrm{m}-$ c) $16.10^{-8} \Omega \mathrm{m}-$ d) $11.10^{-8}$ $\Omega \mathrm{m}]$, compared to $\rho / 2 \mathrm{a}$ and to experimental values. a) hard tin (calculation) and bright tin (experimental) b) soft tin (calculation) and matte tin (experimental).

\section{Discussion and conclusion}

The values calculated from the electro-mechanical model are compared to those measured experimentally. Several observations can be made. The calculated values have the same order of magnitude as the experimental ones. For thicknesses comprised between 1 and $10 \mu \mathrm{m}$ a fairly good agreement is found between the experiment and the model when taking low values of resistivity for the tin layer. The agreement between the experiment and the result of the calculations is better for the soft and matte tin layers. For very thin tin layers $(0.1$ $\mu \mathrm{m}$ to $1 \mu \mathrm{m})$ experimental values are much higher than the calculated ones. The calculations were done for no tin layer $(\mathrm{e}=0)$. No experimental value could be measured for this case since $\mathrm{CuZn}$ without any tin layer is prone to severe oxidation and thus $R c$ values measured would not correspond to the bare $\mathrm{CuZn}$ case. 
No calculation was done for the very thin layers $(0.1$ to $1 \mu \mathrm{m})$ but the calculated variations must be continuous. The measured high values of Rc are thus difficult to account for. Several questions can be raised. We have supposed that the resistivity of the Sn layers was constant for a given finish, whatever the film thickness. Surface analysis has shown the presence of an oxide film on the Sn surface. The ratio of the tin oxide thickness over the tin total thickness can have an effect on the resistance value. The assumption of perfect adhesion of the tin layer on the substrate could also be too simple. In any case it seems that the geometric effect (increase of the contact surface) is stronger than the electrical effect of having a less conducting layer in the interface.

Experimental data show that a nickel underlayer changes the contact behaviour (Fig. 1). To understand the mechanism involved, the influence of such an underlayer was simulated. Results not reported here show that, despite a high yielding stress, the nickel has no visible influence on the contact area. A closer look at some results obtained without the nickel layer explains why this lack of effect is consistent with the model used. Figure 10 displays the vertical plastic deformation $\varepsilon_{\mathrm{pzz}}$ (compression) along the z-axis, for increasing values of the applied force, in the case of a $2 \mu \mathrm{m}$ thick Sn-layer.

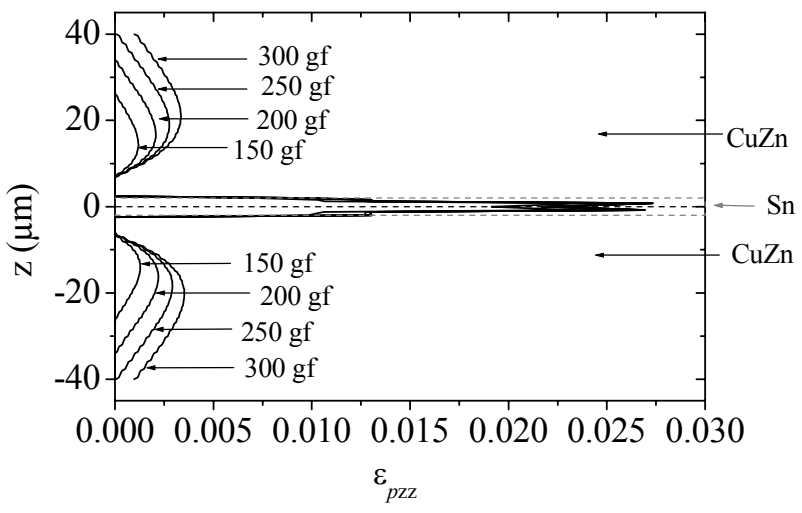

Figure 10: Compression plastic deformation $\varepsilon_{\mathrm{pzz}}$ along the symmetry axis (Sn thickness $=2 \mu \mathrm{m})$.

The plastic deformation appears first in the Sn layer and later in the substrate, about $5 \mu \mathrm{m}$ below the interface. For the considered range of applied force, there is no plastic deformation just under the Sn-layer, and thus adding a material with a high yielding stress at this place has no mechanical influence. The Ni resistivity being about the same as the $\mathrm{CuZn}$ one, the presence of this material has also no electrical influence.

These results lead us to question an important point of the mechanical model: the adhesion of the $\mathrm{Sn}$ layer on the $\mathrm{CuZn}$ substrate was assumed to be perfect, but since strong shearing was calculated to occur at the interface, this assumption might not be satisfied. If the model includes a possible relative sliding at the 
interface, the mechanical behaviour of the contact changes, leading to larger contact area, thinner Sn layer after deformation, and hence lower contact resistance by geometric effect. A nickel underlayer is bound to strongly affect the behaviour of the interface, and this may be the mechanism which explains its influence on the contact. Calculations have been started to explore this explanation, but results are not available yet.

\section{References}

[1] Slade, P., Electrical contacts: principle and applications, M. Dekker, 1999.

[2] Tangena, A.G., The correlation between mechanical stresses and wear in a layered system, Wear, 121, pp. 27-35, 1988.

[3] Noël, S., Lécaudé, N., Correia, S., Gendre, P., Grosjean, A., Electrical and tribological properties of tin plated copper alloy for electrical contact in relation to intermetallic growth, Proc. 52ième IEEE Holm Conference, Montreal, Canada, pp.274-280, pp. 1-10, Sept. 2006.

[4] Noël, S., Lecaudé, N., Alamarguy, D., Boyer, L., Friction properties of perfluorinated polyethers for hot-dipped tin low level separable electrical contacts, Synthetic Lubrication, (18), pp. 179-189, oct.2002.

[5] Ansys website : www.ansys.com

[6] Hammam, T., Friction, wear and electric properties of tin-coated tin bronze for separable electric connectors, Proc $42^{\text {nd }}$ IEEE Holm Conf. On Elec. Contacts, Chicago, USA, pp. 321-330, 1996.

[7] Holm, R., Electric contacts theory and application, Berlin, Germany: Springer-Verlag, 1976. 